\title{
The Timing of Morphogenetic Events in the Regenerating Forelimb of the Axolotl, Ambystoma mexicanum ${ }^{1,2}$
}

\author{
PATRICK W. TANK ${ }^{3}$ \\ Department of Anatomy, The University of Michigan, Ann Arbor, Michigan 48109
}

Received August 23, 1976; accepted in revised form December 23, 1976

\begin{abstract}
The timing of morphogenetic events in the regenerating forelimb of the axolotl was investigated by rotation of limb coverings at well-defined stages in the regenerative process. Both the skin covering the stump and the epidermis covering the regenerate were manipulated independently and together as a unit. The results show that the transmission of morphogenetic information covers a broad range of regenerative stages. This morphogenetic information seems first to become irreversibly fixed in the regenerate by the stage of late bud. The regenerate is sensitive to stump influences at early stages of regeneration, but it becomes insensitive to stump influences by the stage of palette. Evidence is presented which implies that epidermis that covers the regenerate is capable of influencing morphogenesis.
\end{abstract}

\section{INTRODUCTION}

The regeneration of multiple limbs in urodeles following the experimental manipulation of the skin covering the limb stump has been well documented in Triturus cristatus (Droin, 1959; Rahmani, 1960), in Notophthalmus viridescens (Glade, 1957; Settles, 1967, 1970), in Pleurodeles waltl (Lheureux, 1972, 1975a, b), and in Ambystoma mexicanum (Carlson, 1974, 1975b). Deep tissues of the stump have also been shown to exert morphogenetic controls on the regeneration blastema in $N$. viridescens (Glade, 1963), in $P$. waltl (Lheureux, 1972, 1975a, b), and in $A$. mexicanum (Carlson, 1975a, b). Considerably less work has been done to evaluate the role played by epidermis in the morphogenetic process (Glade, 1963; Carlson, 1975a, b). Recent attempts have been made to explain the morphogenetic interactions oc-

1 Taken from a $\mathbf{P h}$. D. dissertation at the University of Michigan.

${ }^{2}$ Supported by NIH Training Grant 5-TOl-GM 00312 and a grant from the Muscular Dystrophy Association to B. M. Carlson.

${ }^{3}$ Present address: Center for Pathobiology, University of California at Irvine. Irvine, California 92717. curring between the amputated stump and the developing regenerate (Carlson, 1975b; Iten and Bryant, 1975), but no work that has been presented previously has examined the timing of morphogenetic events during the process of regeneration.

This paper describes experiments which were designed to answer three basic questions about morphogenetic processes in the regenerating urodele limb: (1) When, during the process of regeneration, are morphogenetic signals transmitted, received, and expressed? (2) To what extent does the skin of the stump influence the regeneration blastema? (3) Can the epidermis overlying the regenerate influence morphogenetic events?

\section{MATERIALS AND METHODS}

All of the experiments reported here utilized the forelimbs of larval axolotls ( $\mathrm{Am}$ bystoma mexicanum). Animals ranged from 85 to $120 \mathrm{~mm}$ in length. Some of the animals were raised in the laboratory at the University of Michigan. The remainder was kindly donated by the axolotl colony of Indiana University. The animals were fed daily on beef liver, and they were maintained in individual plastic containers.

Copyright $(1977$ by Academic Press, Inc. All rights of reproduction in any form reserved.
ISSN 0012-1606 
During critical periods of regeneration, all animals were kept in the dark in a Freas 815 low-temperature incubator to maintain a constant temperature of $21^{\circ} \mathrm{C}$. At other times, the animals were maintained at $21 \pm 2^{\circ} \mathrm{C}$ under a 12-hr light cycle.

Experimental animals were prepared by a variety of surgical techniques that can best be described in conjunction with the results. Prior to surgery, all animals were anesthetized in ethyl $\mathrm{M}$-aminobenzoate methanesulfonate (Eastman), diluted 1:1000 in tap water. Selected limbs from all groups of series 1 and 2 were fixed in Bouin's solution. These regenerates were serially sectioned at $7 \mu \mathrm{m}$ and stained with Ehrlich's hematoxylin and eosin as controls for the staging of regenerates.

All experimental regenerates were prepared as whole mounts and stained for cartilage by the Victoria blue B method of Bryant and Iten (1974). This staining technique permitted the analysis of regenerates on the basis of skeletal morphology.

\section{RESULTS}

(I) When, during the Regenerative Process, Are Morphogenetic Signals Transmitted, Received, and Expressed?

The goal of the first two series of experiments was to determine a range of stages in the regenerative process during which morphogenetic events are occurring. In order to study the timing of morphogenetic events, a morphogenetic stimulus of known duration was supplied to the regenerate by rotation of the coverings of the stump and regenerate at precise times in the regenerative process.

Series 1 . The 72 axolotls of series 1 comprised four experimental groups. All experimental animals underwent surgery for $180^{\circ}$ rotation of the skin overlying the upper forelimb, followed by amputation through the cuff of rotated skin (Fig. 1). Limbs were allowed to regenerate in the presence of the rotated skin. At each of four stages of regeneration, one group underwent surgery for rerotation of the pre-

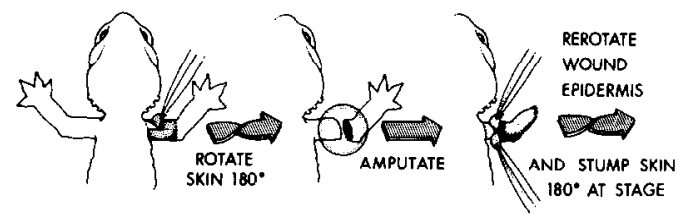

FIG. 1. Methodology of series 1. Skin cuffs were removed, followed by amputation through the cuff. At each of four stages of regeneration (DD, MB, LB, Pal), both the stump skin cuff and the epidermis covering the regenerate were rerotated.

viously rotated cuff of skin and the epidermis covering the regenerate, returning the skin to its original position. This was accomplished by making a skin incision at the proximal border of the previously rotated cuff, followed by loosening of the skin and epidermis of the regenerate with bent microdissecting forceps. The cuff and epidermis were then peeled off much as one would remove a sock by turning it inside out. After turning this "sock" right side out, it was replaced over the stump and blastema and was then rotated $180^{\circ}$. Two proximal sutures secured each cuff. The stages at which rerotation surgery was performed were: (i) stage of dedifferentiation (DD); (ii) stage of medium bud (MB); (iii) stage of late bud (LB); (iv) stage of palette (Pal) (Tank et al., 1976). The second surgery removed the disharmony of the rotated skin and epidermis at a clearly defined point in the regenerative process. Sham-surgical controls were prepared for each group by $0^{\circ}$ rotation prior to amptutation through the graft. At the appropriate stages of regeneration, the controls underwent surgery for $0^{\circ}$ rerotation of the cuff of skin and the epidermis covering the regenerate.

The results of this series of experiments are summarized in Table 1. Mature regenerates were grouped under four classifications. Normal (Nor) limbs were limbs complete with four digits of normal morphology (Fig. 2). Supernumerary (Sn) regenerates were those possessing greater than the normal complement of four digits (Fig. 3 and 4). Abnormal (Abn) limbs were limbs bearing four-digit hands of grossly 
TABLE 1

Series 1: Rotation of Stump Skin Cuff at Day 0 and Rerotation of Stump Skin Cuff and Regenerate Epidermis at Various Stages

\begin{tabular}{|c|c|c|c|c|c|c|}
\hline & \multicolumn{5}{|c|}{ Number of limbs } & \multirow{2}{*}{$\begin{array}{l}\text { Mean number } \\
\text { of Sn digits }\end{array}$} \\
\hline & Valid & Nor $^{a}$ & Abn & Нyро & Sn & \\
\hline \multicolumn{7}{|l|}{ Experimental } \\
\hline Group 1 (DD) & 21 & 15 & 0 & 1 & $5(23.8)^{b}$ & 5.0 \\
\hline Group $2(\mathrm{MB})$ & 23 & 17 & 0 & 4 & $2(8.7)$ & 5.0 \\
\hline Group 3 (LB) & 13 & 3 & 0 & 0 & $10(76.9)$ & 6.6 \\
\hline \multirow[t]{2}{*}{ Group 4 (Pal) } & 18 & 0 & 4 & 1 & $13(72.2)$ & 6.5 \\
\hline & & & & & & Normal (\%) \\
\hline \multicolumn{7}{|l|}{ Control } \\
\hline Group 1 (DD) & 4 & 4 & 0 & 0 & 0 & 100.0 \\
\hline Group 2 (MB) & 4 & 3 & 0 & 1 & 0 & 75.0 \\
\hline Group 3 (LB) & 2 & 1 & 0 & 0 & 1 & 50.0 \\
\hline Group 4 (Pal) & 9 & 2 & 5 & 2 & 0 & 22.2 \\
\hline
\end{tabular}

a Abbreviations: Nor, limbs complete with four digits of normal morphology; Abn. limbs bearing fourdigit hands of grossly abnormal morphology; Hypo, limbs possessing fewer than the normal complement of four digits; and $\mathrm{Sn}$, limbs possessing greater than the normal complement of four digits.

${ }^{b}$ Numbers within parentheses indicate percentages of supernumerary limbs present in the experimental group.

abnormal morphology (Fig. 5). Hypomorphic (Hypo) limbs were limbs possessing fewer than the normal complement of four digits (Fig. 6). In addition, the percentage of supernumerary limbs present in the experimental group was calculated, as was the percentage of normal limbs in the control group. The mean number of digits present on the supernumerary limbs was also computed.

Regenerates produced under a minimal duration of exposure to rotated stump skin, as accomplished by rerotation of cuff and epidermis at the dedifferentiation stage of regeneration (Table 1), show both a low percentage of supernumerary limbs and a low mean number of digits on those supernumerary limbs. Those multiple structures that did form were very simple in morphology. Exposure of the regenerate to a slightly longer period of disharmonic stimuli, as done in group 2 in which rerotation was performed at the medium bud stage of regeneration, produced a lower percentage of supernumerary limbs. The mean number of digits on those supernumerary limbs was the same as that produced in group 1. In both groups 1 and 2 , most sham-controls were normal, the only abnormality being a hypomorphic twodigit regenerate.

As exposure to the disharmonic information provided by rotated stump skin was lengthened by rerotation at the late bud stage, a pronounced increase in both the percentage of supernumerary limbs formed and in the mean number of digits on those supernumerary limbs occurred (Table 1, group 3). When rerotation was performed at the palette stage (group 4), the results obtained were almost identical to those seen in group 3 . No normal regenerates were produced in this experiment, and the supernumerary regenerates formed were of moderate complexity. Sham controls for groups 3 and 4 were seldom normal, but in only one case was a supernumerary regenerate seen. This limb produced a four-digit regenerate which arose from the ventral surface of the elbow near the junction between the epidermis and the whole skin. The other control regenerates were abnormal or hypomorphic, indicating that the trauma of surgery was not sufficient to cause the high percentages of supernumerary regenerates seen in this series.

Histological controls for all groups of se- 

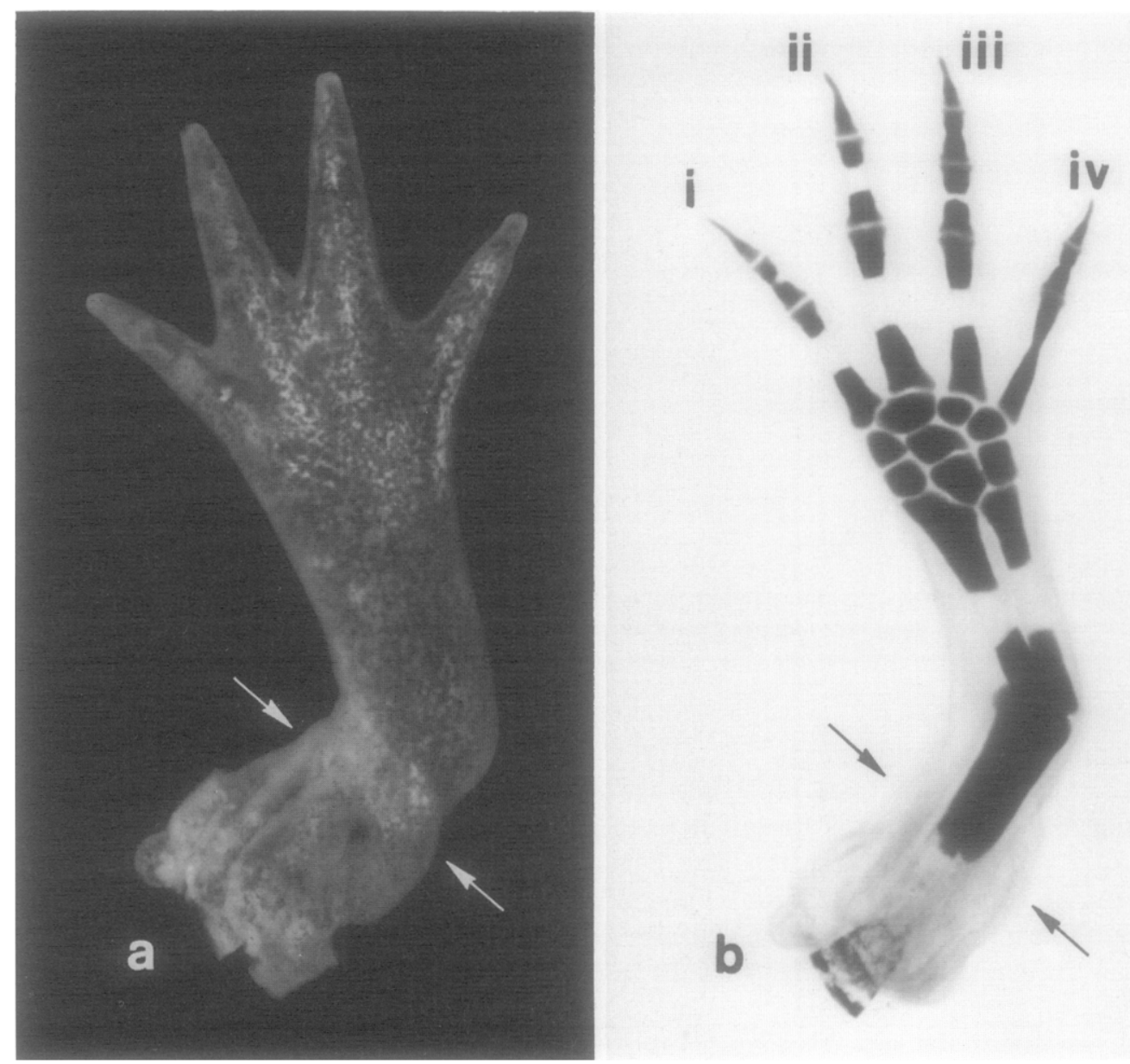

Fig. 2. A normal four digit regenerate. Arrows indicate the level of amputation. Lateral view; dorsal is to the right. $9 \times$. (a) Gross appearance 80 days after amputation. Note the smooth texture of the skin covering the regenerated part. (b) Skeletal morphology. The skeletal formula is 1:2:8:4:9. Roman numerals indicate digits numbered from the radial side of the hand. Digit III is the only digit normally bearing three phalanges. Victoria blue skeletal stain.

ries 1 verified the stages at which rerotation surgery was performed. In addition, a histological study of 20 cuffs of skin removed from upper forelimbs by blunt dissection revealed a clean plane of separation between the dermis and underlying muscle. Contamination of skin grafts by skeletal muscle was never observed.

The results of series 1 indicate a direct relationship between the duration of the disharmonious influence and the frequency of multiple regeneration. Removal of the disharmonious stimulus prior to the late bud stage allows the blastema to regu- late and correct for misinformation received before this time. Therefore, the disharmonious morphogenetic information first becomes irreversibly fixed in the regenerate between the stages of medium bud and late bud.

Series 2. The second series of experiments used to explore the timing of morphogenetic events in the regenerating limb was the converse of series 1 . All 69 axolotls of series 2 had both forelimbs amputated without previous surgical manipulations (Fig. 7). At each of the four stages of regeneration mentioned previously, one 
group of animals underwent surgery for $180^{\circ}$ rotation of the cuff of skin covering the upper forelimb and the epidermis covering the regenerate. The surgery in this experiment introduced a morphogenetic stimulus of known duration in the regenerative process. Sham-operated controls were again prepared. Limbs of control animals were amputated as above, followed by $0^{\circ}$ sham rotations of the skin covering the stump and the epidermis covering the regenerate, at the appropriate stages.

The results of series 2 are presented in Table 2. When a disharmonious influence was supplied to the regenerating system at the stage of dedifferentiation and was allowed to exist for the remainder of regeneration (as was done in group 1 of series 2), a high percentage of supernumerary limbs was produced. These supernumerary regenerates also possessed a high mean number of digits, indicating their complexity.

When the disharmonious influence of rotated skin and wound epidermis was supplied at the stage of medium bud (as was done in group 2 of series 2), a slightly higher percentage of supernumerary regenerates was produced. A high mean number of digits was formed on these supernumerary regenerates of group 2 . There were no normal regenerates produced in this group, and, as in group 1, digital counts were generally high. In one instance, a 16-digit regenerate was produced (Fig. 8).

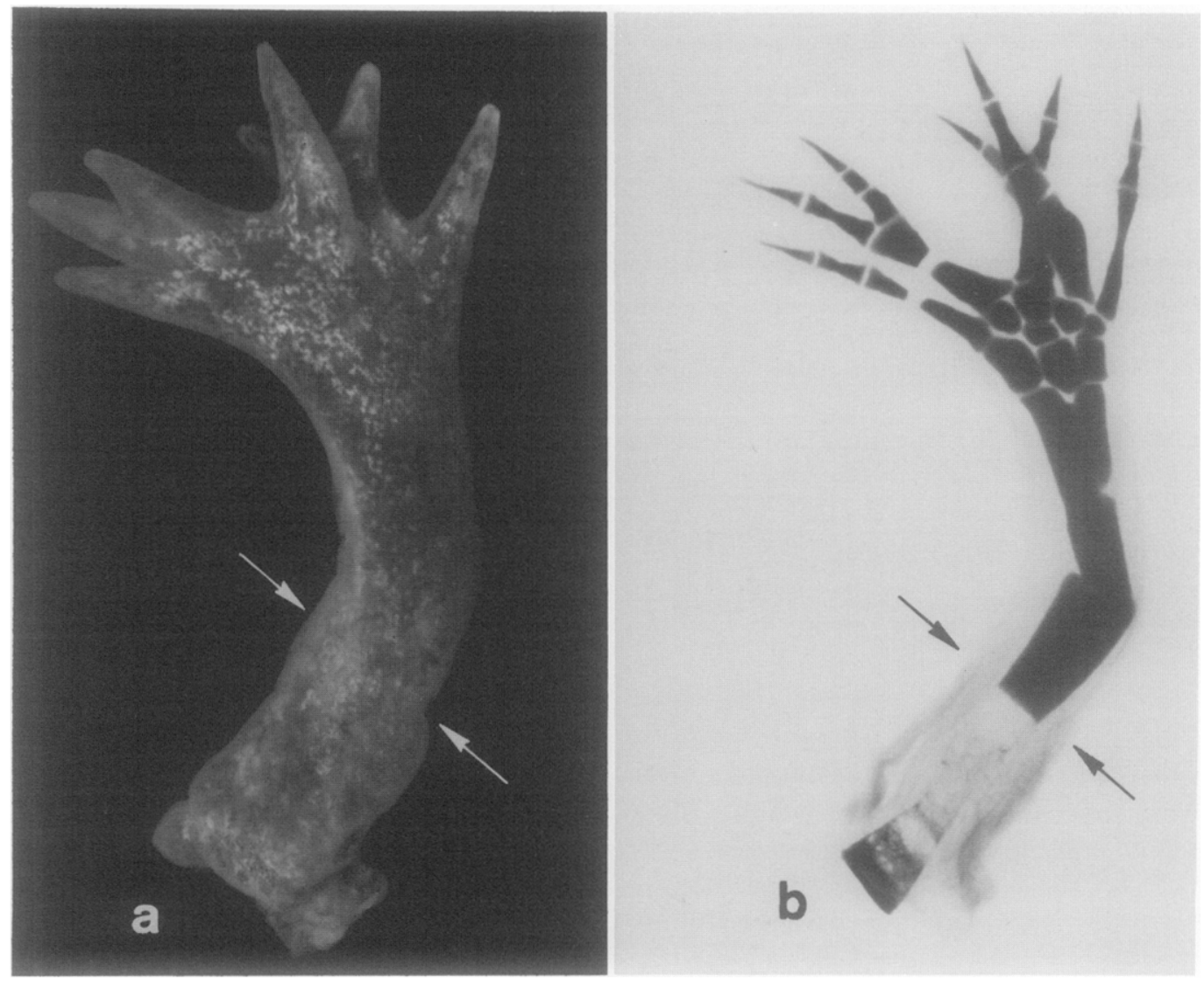

FIG. 3. A triaxial supernumerary regenerate. Arrows indicate the level of amputation. Lateral view; dorsal is to the right. $9 \times$. (a) Triaxial regenerates bear digits in three conjoined planes. Note the overlapping of digits in this specimen. (b) The skeletal morphology recapitnlates the overlapping character of this regenerate. Victoria blue skeletal stain. 

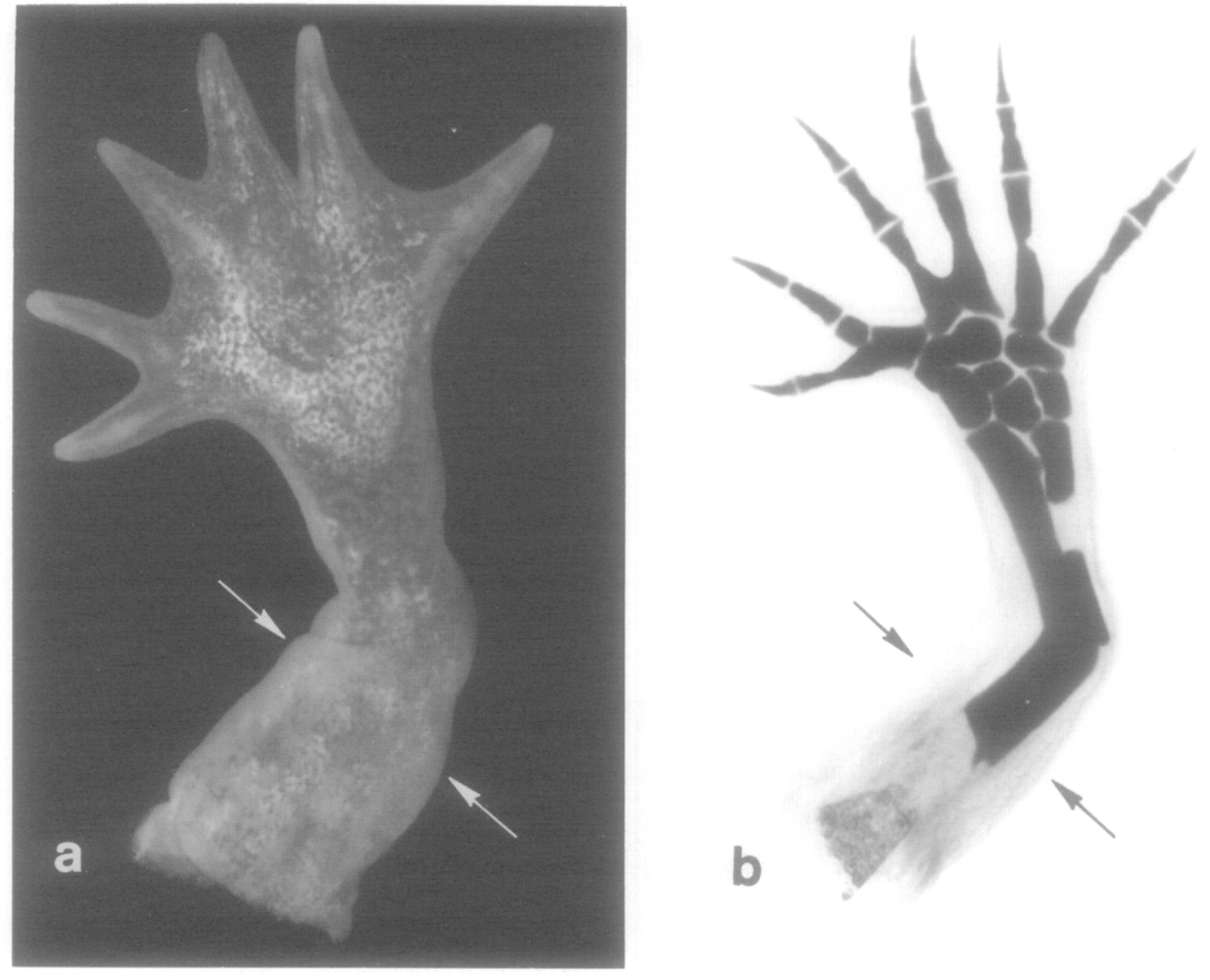

FIG. 4. A planar regenerate. Arrows indicate level of amputation. Lateral view: dorsal is to the right. 9 $x$. (a) Gross appearance. All digits lie in the same plane. (b) Skeletal morphology. No structures in either the carpal or digital regions lie outside of the plane of the hand. Victoria blue skeletal stain.

When the disharmonious influence was supplied at the late bud stage of regeneration (group 3 of series 2) and was allowed to remain for the duration of regeneration, a slight increase in the percentage of supernumerary limbs resulted, and a larger increase in the mean number of digits produced on those limbs occurred. The regenerates of group 3 were very complex in structure. When the disharmonious influence was supplied at the palette stage (group 4 of series 2) and was allowed to exist for the remainder of regeneration, a slightly lower percentage of supernumerary regenerates was produced, and a lower mean number of digits developed in those supernumerary regenerates. There were only two normal regenerates among the seven sham-operated controls prepared for group 4. The remaining five developed abnormal four-digit regenerates. However, supernumerary structures never formed on sham-control limbs in any of the groups of series 2 . This indicates that the trauma of surgery was not sufficient to cause the formation of supernumerary limbs. All histological controls for these groups demonstrated the correctness of identification of the stages at which rotations were performed.

The results of series 2 indicate that multiple regenerates are produced, regardless of the stage at which rotation of limb coverings is performed. Therefore, the regenerate is sensitive to morphogenetic input throughout the range of stages tested.

In summary, the results of series 1 and 2 reveal that the passage of morphogenetic 
information is not a single pinpoint event in the regenerative process. Information is transmitted, received, and expressed at least from the end of the stage of medium bud (at which time irreversible specification begins) through the stage of palette. If significant morphogenetic information is present in the early blastema, it does not appear to become stabilized until late in the stage of medium bud or early in the stage of late bud.

(II) To What Extent Does the Skin of the Stump Influence the Regeneration Blastema?

The experiments performed in series 3 and 4 were aimed at determining the effect of rotating only the skin of the stump, independent of the epidermis covering the regenerate.

Series 3. In series 3 , the skin of the upper forelimbs of 43 experimental animals was rotated $180^{\circ}$, followed by amputation through the graft. At each of two stages of regeneration ( $\mathrm{MB}, \mathrm{Pal}$ ), the influence of the rotated skin was removed by rerotation of the cuff of stump skin by $180^{\circ}$ (Fig. 9). The epidermis covering the regenerate was not disturbed. The second surgery sucessfully eliminated the disharmony between the tissues of the stump without mechanical trauma to the regenerate. Sham-surgical controls were prepared by $0^{\circ}$ rotation of skin prior to ampu-
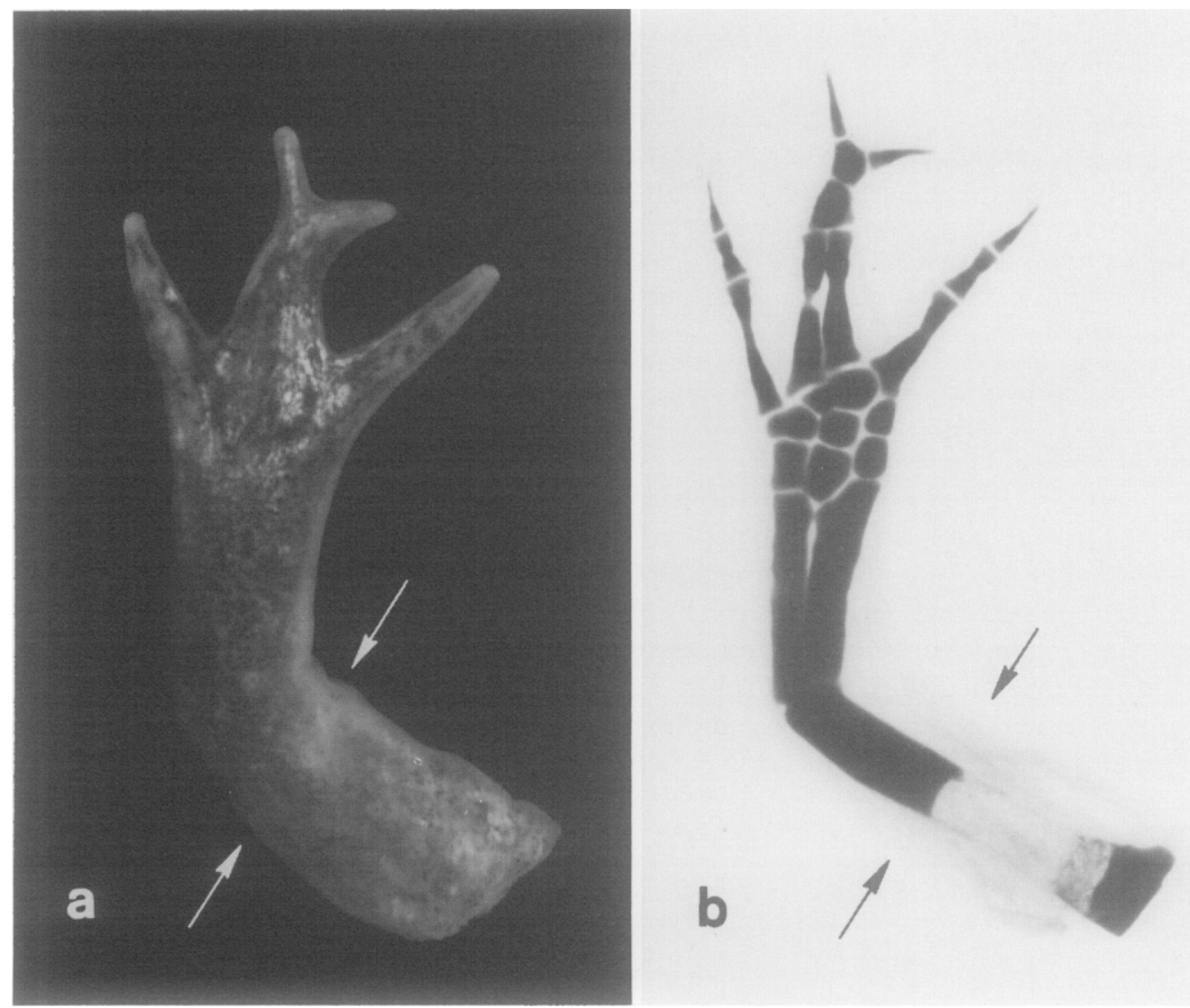

FIG. 5. An abnormal four digit regenerate. Arrows indicate the level of amputation. Lateral view; dorsal is to the left. $9 \times$. (a) Gross appearance. Abnormal regenerates do not always produce digits in one plane as this specimen might indicate. (b) Skeletal morphology. This specimen shows a fusion of phalangeal elements of digits II and III. Deletions and duplications of skeletal elements are also common in abnormal regenerates. Victoria blue skeletal stain. 


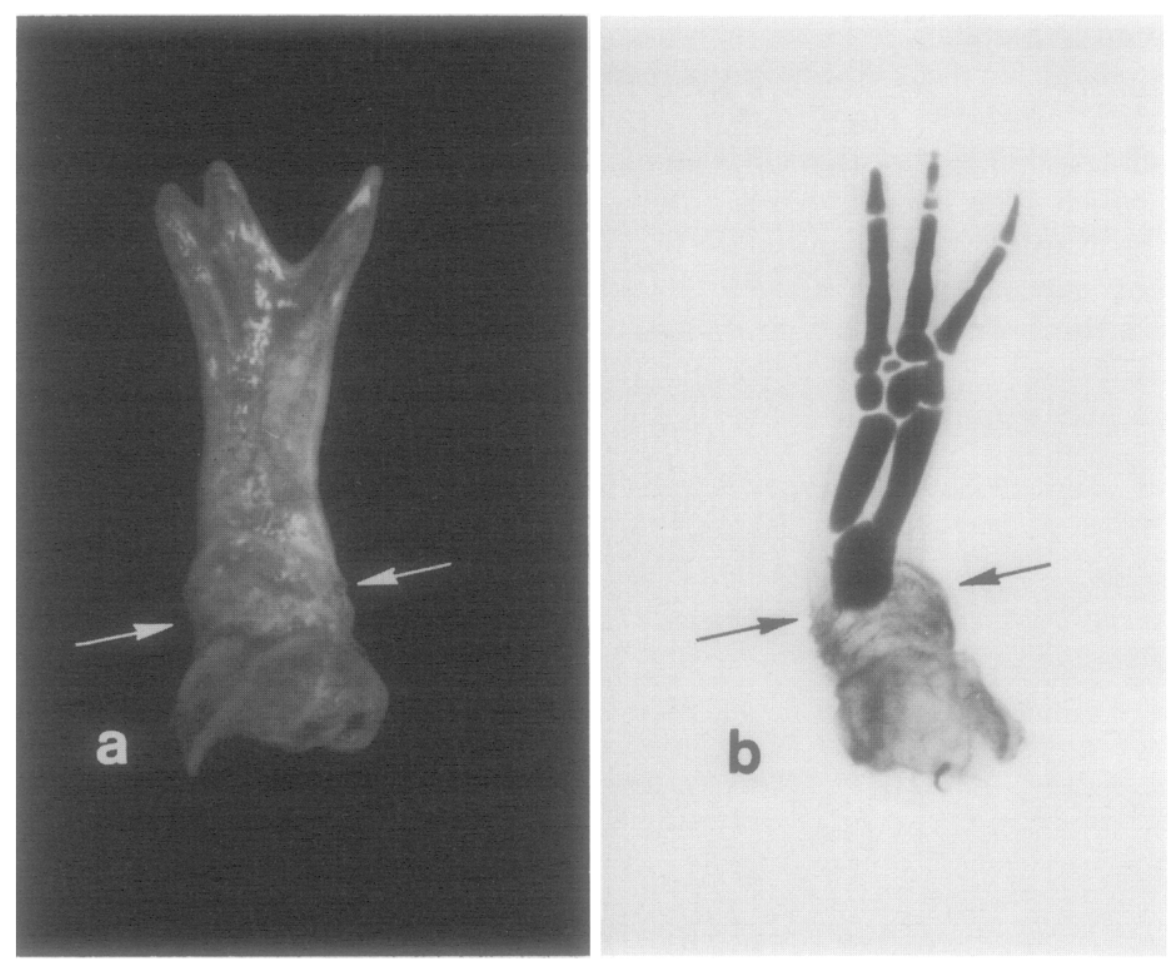

FIG. 6. A hypomorphic regenerate. Arrows indicate the level of amputation. Lateral view; dorsal is to the left. $9 \times$. (a) Gross appearance. Three- and two-digit hypomorphic regenerates are produced in equal proportions. Hypomorphic spikes are seldom seen. (b) The skeletal morphology indicates several deletions of skeletal elements. Fusions are also very common in hypomorphic regenerates. Victoria blue skeletal stain.

tation through the graft, followed by $0^{\circ}$ rerotation of only the skin covering the stump at each of the two stages.

The results of series 3 appear in Table 3 . When the disharmonic influence supplied by rotated skin was removed by rerotation of the cuff of skin at the medium bud stage, the percentage of supernumerary regenerates obtained was high (Table 3, group 1). These regenerates were complex in structure. When the disharmonious influence of rotated stump skin was removed at the palette stage by rerotation (Table 3 ), high percentages of complex regenerates were again produced. The percentage of supernumerary limbs produced is similar to the percentage of supernumerary limbs regenerated after $180^{\circ}$ skin cuff rotation without subsequent rerotation. One regenerate of group 2 developed three hands (Fig. 10). All sham-operated controls pre-

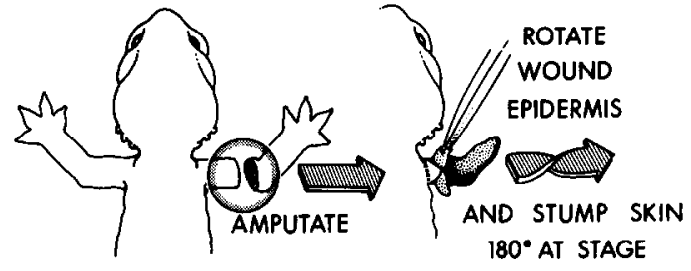

Fig. 7. Methodology of series 2. Limbs were amputated without previous surgery. At each of four stages of regeneration (DD, MB, LB, Pal), both the stump skin and the epidermis covering the regenerate were rotated.

pared for the two groups of series 3 regenerated normally.

Based on the results of series 3, it can be concluded that the rotation and rerotation of the skin covering the stump has a pronounced effect on the morphology of the regenerate, even though the epidermal covering of the regenerate was left undisturbed. The percentages of supernumerary 
TABLE 2

Series 2: Rotation of Stump Skin Cuff and Regenerate Epidermis at Various Stages

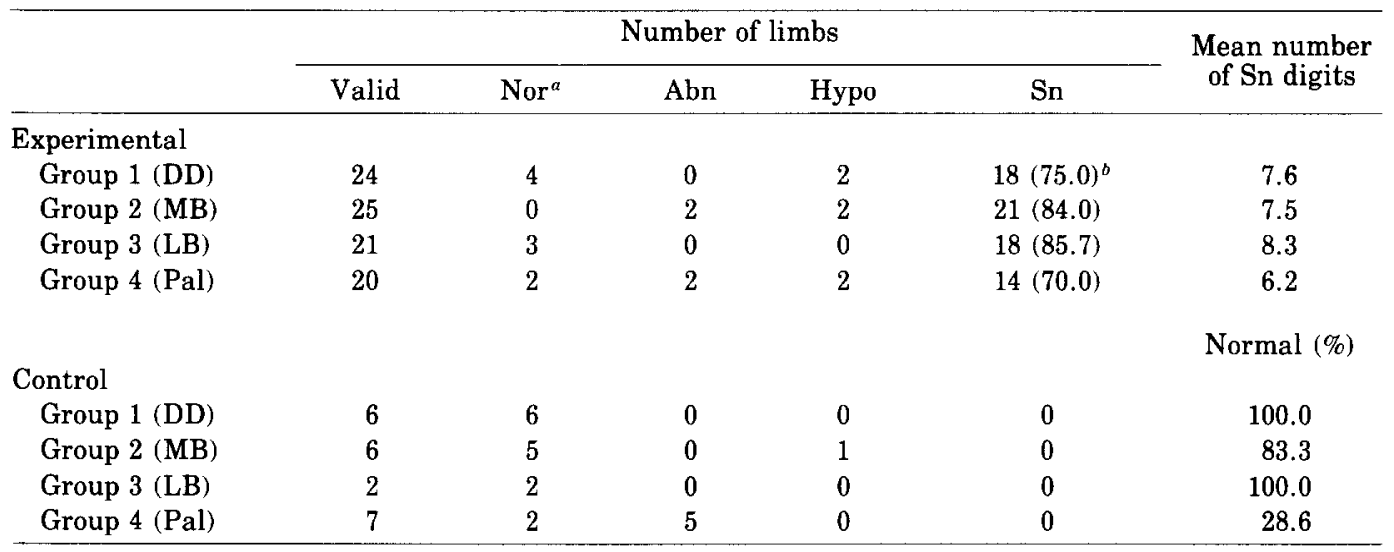

a Abbreviations are those in footnote $a$ of Table 1.

${ }^{b}$ Numbers within parentheses indicate percentages of supernumerary limbs present in experimental group.

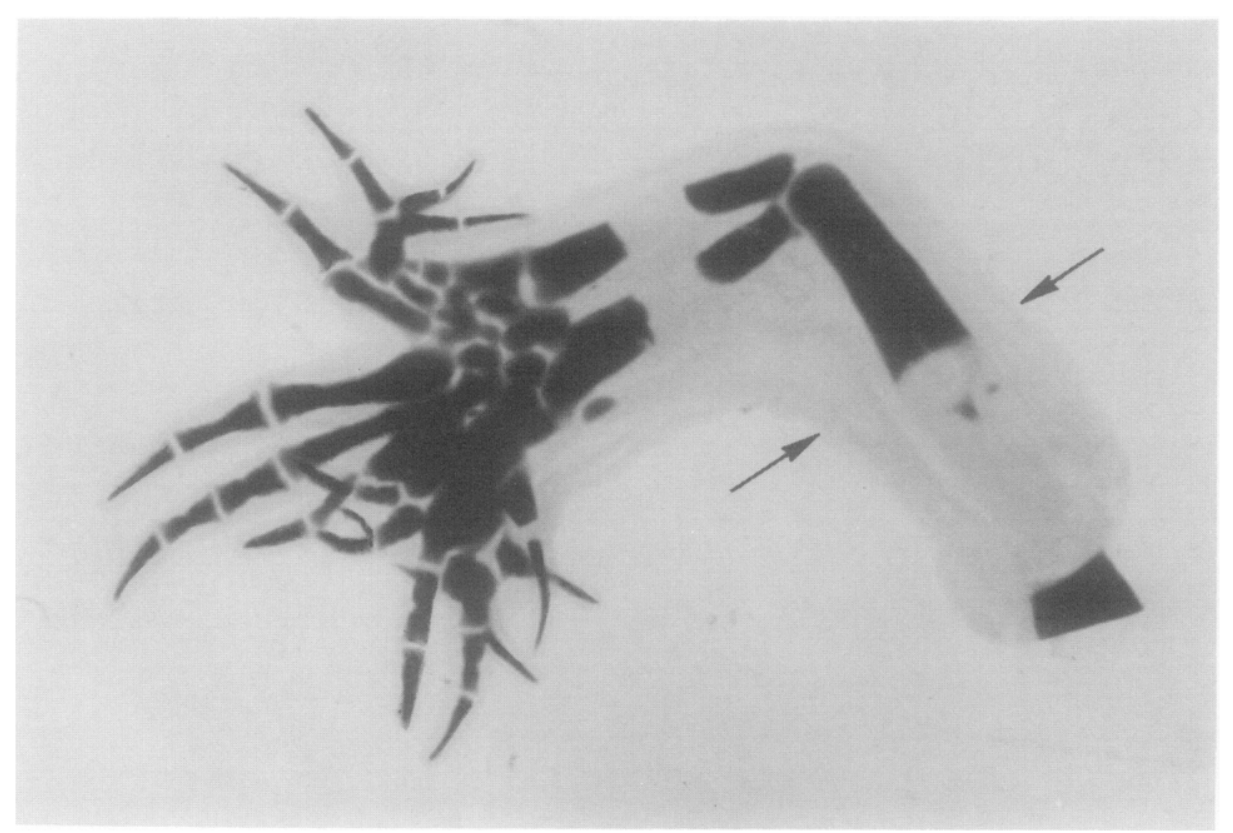

FIG. 8. A 16-digit regenerate produced in group 2 of series 2 . It is the most complex regenerate produced in any of the experiments reported in this paper. Arrows indicate the level of amputation. Lateral view; dorsal is upward. Victoria blue skeletal stain. $9 \times$.

regenerates formed and the complexity of these regenerates are comparable to the results obtained from control groups bearing $180^{\circ}$-rotated skin cuffs.

Series 4 . Series 4 is the converse experiment to series 3 . In series 4 , all forelimbs of the 20 experimental animals were am- putated without previous surgical manipulations (Fig. 11). At each of two stages of regeneration (stages of medium bud and palette), only the skin covering the stump of the limb was rotated $180^{\circ}$; the epidermis of the regenerate remained undisturbed. The second surgery introduced a morpho- 
genetic influence of stump origin at a clearly defined point in the regenerative process. Sham-surgical controls were prepared by amputation of unoperated limbs, followed by $0^{\circ}$ sham rotations of the skin covering the stump at the stages of medium bud and palette.

The results of series 4 are summarized in Table 4. When only the stump skin was rotated at the medium bud stage of regeneration, leaving the epidermis of the regenerate undisturbed, a high percentage of supernumerary regenerates developed. These regenerates were of high complexity, as evidenced by their high average number of digits (Table 4, group 1). When the disharmonious influence was provided at the palette stage by rotation of stump skin independent of the cpidermis covering the regenerate, a very low percentage of supernumerary limbs was produced (Table 4 , group 2 ). The only supernumerary

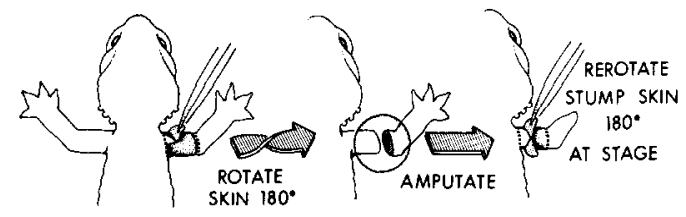

Fig. 9. Methodology of series 3. Cuffs of stump skin were rotated $180^{\circ}$ before amputation. At each of two stages of regeneration (MB, Pal), the rotated cuffs were rerotated. The wound epidermis was not manipulated. regenerate that did form in this group was a rather unusual supernumerary forearm and hand which arose from the mid-forearm level of the normally regenerated limb, rather than from the wrist or hand region as is the usual case (Fig. 12). Shamsurgical controls prepared for both groups of series 4 regenerated normally. The results of this experiment indicate that the regenerate is still receptive to morphogenetic influences emanating from the limb stump at the stage of medium bud. The regenerate is largely insensitive to stump influences at the stage of palette.

In summary, the results of series 3 and 4 indicate that the skin covering the stump of the amputated limb has a great deal of influence over the morphology of the regenerate at early stages of regeneration. However, when a disharmonious morphogenetic influence of stump origin was introduced late in the regenerative process (series 4, group 2), the regenerate was largely insensitive to the stump influence.

\section{(III) Can the Epidermis Overlying the Regenerate Influence Morphogenetic Events?}

Series 5. In series 5, the forelimbs of 15 experimental animals were amputated without previous surgical preparation (Fig. 13). At the stage of medium bud, only the epidermis covering the regenerate was

TABLE 3

Serifs 3: Rotation of Stump Skin Cuff at Day 0 and Rerotation of Stump Skin Cuff at Various STAGES

\begin{tabular}{|c|c|c|c|c|c|c|}
\hline & \multicolumn{5}{|c|}{ Number of limbs } & \multirow{2}{*}{$\begin{array}{l}\text { Mean number } \\
\text { of Sn digits }\end{array}$} \\
\hline & Valid & Nor $^{a}$ & Abn & Hypo & $\mathrm{Sn}$ & \\
\hline \multicolumn{7}{|l|}{ Experimental } \\
\hline Group 1 (MB) & 21 & 2 & 2 & 3 & $14(66.7)^{b}$ & 6.9 \\
\hline \multirow[t]{2}{*}{ Group $2(\mathrm{Pal})$} & 19 & 1 & 2 & 1 & $15(78.9)$ & 6.7 \\
\hline & & & & & & Normal $(\%)$ \\
\hline \multicolumn{7}{|l|}{ Control } \\
\hline Group 1 (MB) & 2 & 2 & 0 & 0 & 0 & 100.0 \\
\hline Group 2 (Pal) & 4 & 4 & 0 & 0 & 0 & 100.0 \\
\hline
\end{tabular}

a Abbreviations are those in footnote $a$ of Table 1.

${ }^{b}$ Numbers within parentheses indicate percentages of supernumerary limbs present in experimental group. 


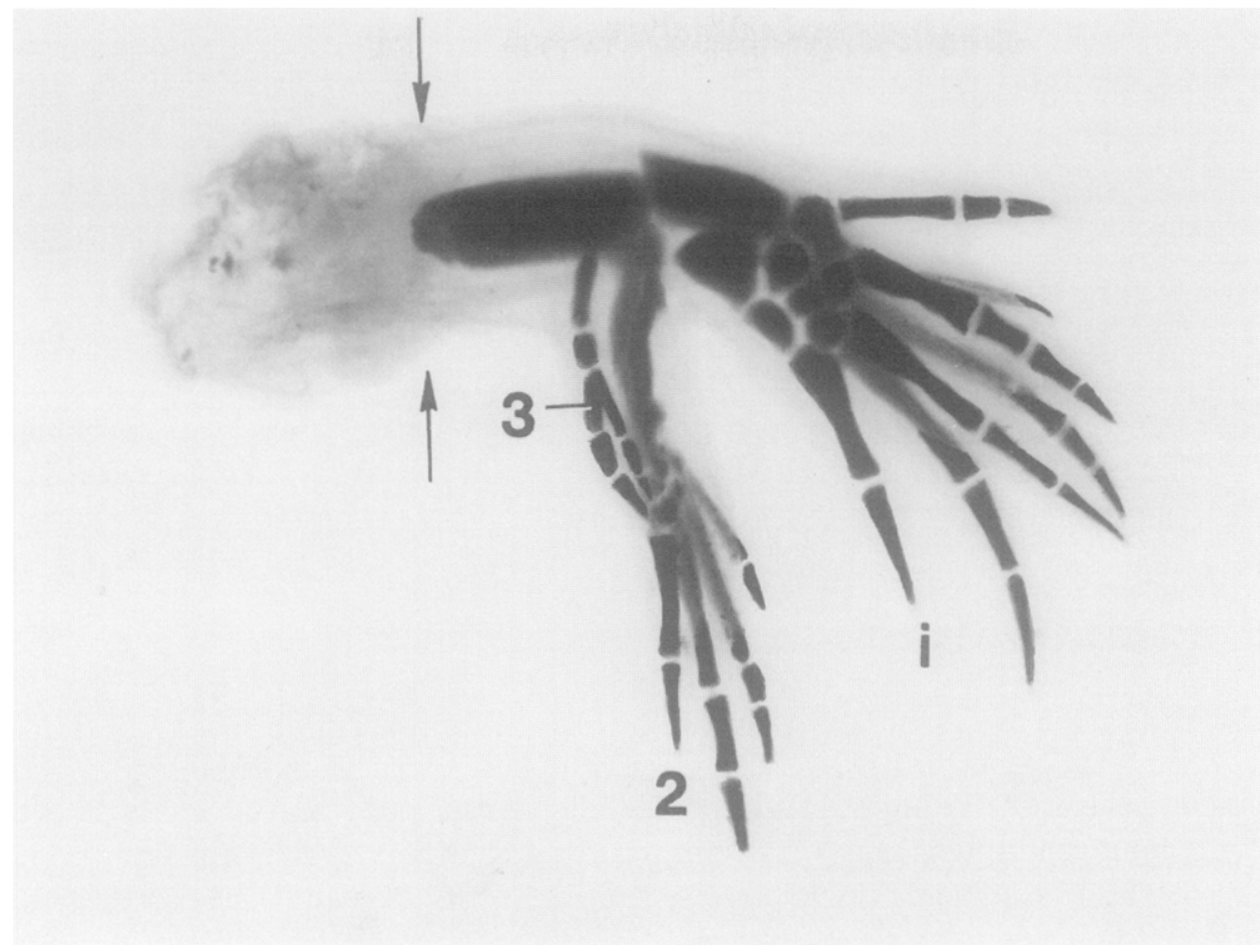

FIG. 10. A supernumerary regenerate from series 3 . This limb displays a mirror image duplication of the normal hand with digit I (i) in common. A second supernumerary limb of normal asymmetry (2) can be seen projecting ventrally from the humerus, perpendicular to the axis of the normal regenerate. A third regenerate bearing two digits (3) accompanies the second. Arrows indicate the level of amputation. Lateral view; dorsal is upward. Victoria blue skeletal stain. $9 \times$.

rotated $180^{\circ}$, leaving the skin covering the stump undisturbed. After mature regenerates had formed, these same forelimbs were reamputated at a more proximal level to allow the rotation of epidermis at the stage of medium bud to be repeated. This process was again repeated to produce rotated cpidermal grafts on regenerates at the stage of palette. Sham-surgical controls were prepared by $0^{\circ}$ epidermal rotations at the stages of medium bud and palette.

The results of series 5 (Table 5) indicate that rotation of the epidermis covering the regenerate does cause the production of multiple regenerates at all stages tested. However, histological analysis of 15 epidermal specimens, remuved by blunt dissection from regenerates at the stages of medium bud and palette, showed that all specimens were contaminated by blaste-

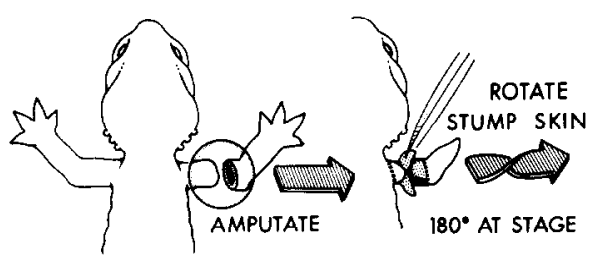

Fic. 11. Methodology of series 4. Forelimbs were amputated without previous surgery. At each of two stages of regeneration (MB, Pal), a cuff of stump skin was rotated $180^{\circ}$. The wound epidermis was not manipulated.

mal cells to varying degrees (Fig. 14). Therefore, it cannot be stated with certainty that the results seen in series 5 were caused by rotated epidermis.

In summary, the results of series 5 indicate that the rotation of either the epidermis covering the regenerate or something adhering to the epidermis is sufficient to cause the production of multiple regenerates. 
TABLE 4

Series 4: Rotation of Stump Skin Cuff at Various Stages of Regeneration

\begin{tabular}{lcccccc}
\hline & \multicolumn{5}{c}{ Number of limbs } & $\begin{array}{c}\text { Mean number } \\
\text { of Sn digits }\end{array}$ \\
\cline { 2 - 5 } & Valid & Nor $^{a}$ & $\Lambda \mathrm{bn}$ & Hypo & $\mathrm{Sn}$ & \\
\hline Experimental & & & & & & \\
Group 1 (MB) & 18 & 5 & 3 & 0 & $10(55.6)^{b}$ & 7.9 \\
Group 2 (Pal) & 17 & 14 & 2 & 0 & $1(5.9)$ & 8.0 \\
& & & & & & $\begin{array}{c}\text { Normal } \\
(\%)\end{array}$ \\
Control & & & & & 0 & 100.0 \\
$\quad$ Group 1 (MB) & 1 & 1 & 0 & 0 & 0 & 100.0 \\
Group 2 (Pal) & 1 & 1 & 0 & 0 & & \\
\hline
\end{tabular}

${ }^{a}$ Abbreviations are those in footnote $a$ of Table 1.

${ }^{b}$ Numbers within parentheses indicate percentages of supernumerary limbs present in the experimental group.

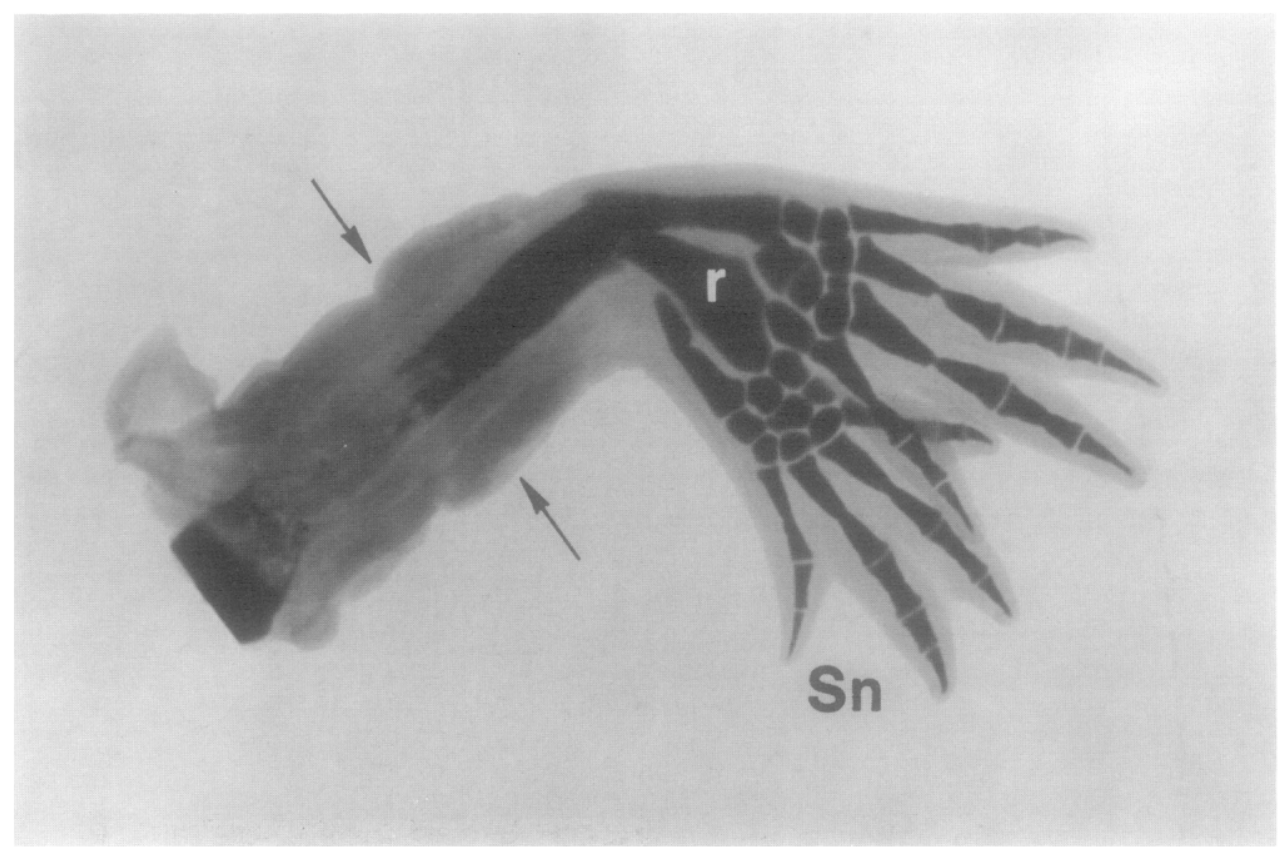

Fig. 12. A complex supernumerary regenerate from series 4 . This regenerate displays a hand of correct asymmetry for the limb stump and a mirror image supernumerary regenerate ( $\mathrm{Sn}$ ). The regenerates share the radius (r), but each possesses its own complete set of carpals. Arrows indicate the level of amputation. Lateral view; dorsal is upward. Victoria blue skeletal stain. $9 \times$.

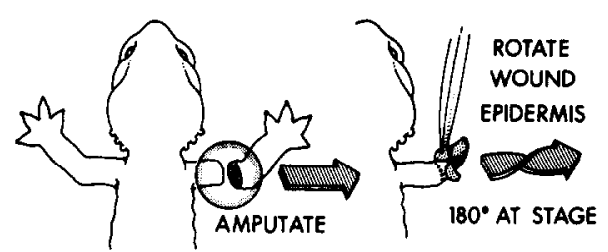

FIG. 13. Methodology of series 5. Forelimbs were amputated without previous surgery. At each of two stages (MB, Pal), the epidermis covering the regenerate was rotated $180^{\circ}$.

\section{DISCUSSION}

As an aid to the discussion, a summary of the techniques and results is presented in Table 6.

The principle findings of the experiments that have been reported here can be simply stated. (1) The information that directs the morphogenesis of the regenerating axolotl limb is transmitted, received, 
TABLE 5

Series 5: $180^{\circ}$ Rotation of the Epidermis Which Covers the Regenerate at Vahious Stages

\begin{tabular}{|c|c|c|c|c|c|c|}
\hline & \multicolumn{5}{|c|}{ Number of limbs } & \multirow{2}{*}{$\begin{array}{c}\text { Mean num } \\
\text { ber of } \mathrm{Sn} \\
\text { digits }\end{array}$} \\
\hline & Valid & Nor $^{a}$ & Abn & Hypo & $\mathrm{Sn}$ & \\
\hline \multicolumn{7}{|l|}{ Experimental } \\
\hline Group 1 (MB) & 18 & 10 & 0 & 0 & $8(44.4)^{b}$ & 5.3 \\
\hline Group 2 (MB) & 22 & 16 & 1 & 0 & $5(22.7)$ & 5.4 \\
\hline \multirow[t]{2}{*}{ Group $3(\mathrm{Pal})$} & 22 & 2 & 2 & 0 & $18(81.8)$ & 5.5 \\
\hline & & & & & & $\underset{(\%)}{\text { Normal }}$ \\
\hline \multicolumn{7}{|l|}{ Control } \\
\hline Group 1 (MB) & 5 & 5 & 0 & 0 & 0 & 100.0 \\
\hline Group 2 (MB) & - & - & - & - & - & - \\
\hline Group 3 (Pal) & 2 & 2 & 0 & 0 & 0 & 100.0 \\
\hline
\end{tabular}

a Abbreviations are those in footnote $a$ of Table 1 .

${ }^{\circ}$ Numbers within parentheses indicate percentages of supernumerary limbs present in experimental group.

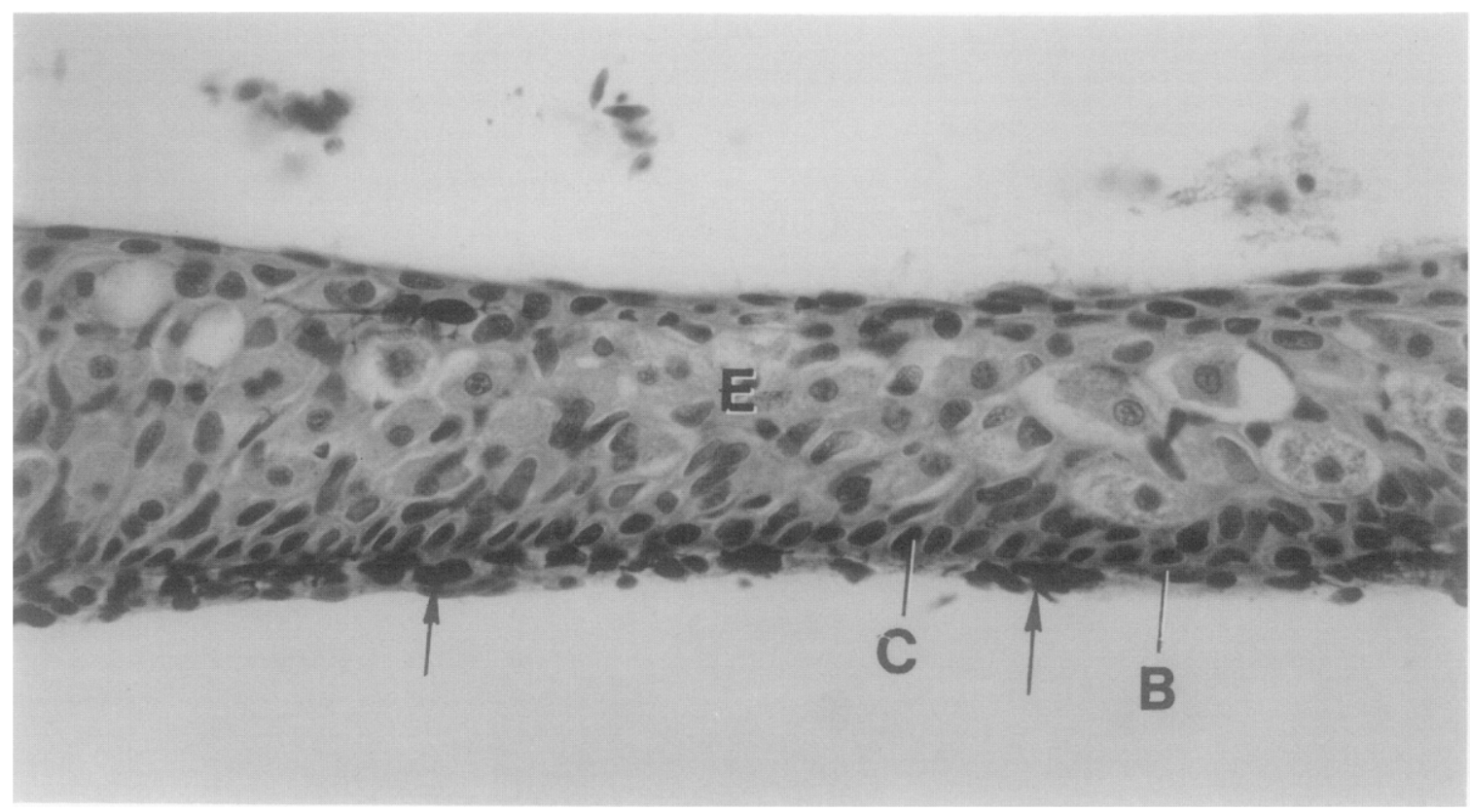

Fig. 14. Histological appearance of an epidermal specimen removed by blunt dissection from a regenerate at the stage of medium bud. The thick epidermis (E) displays a very regular layer of basal cells (C) and a well defined basement membrane (B). Blastemal cells (arrows) adhere to the deep surface. The free edge of the epidermis is upward. H\&E. $430 \times$.

and expressed over a broad range of stages. (2) The morphogenetic information seems first to become irreversibly fixed in the regenerate by the beginning of the late bud stage of regeneration. These results suggest a number of thoughts about the interactions between the stump and the regeneration blästema in the establishment of limb patterns.

There is considerable evidence that the cells of the early blastema may possess morphogenetic information or positional values of the stump regions from which they originated (DeBoth, 1970; Faber, 
TABLE 6

Summary of Techniques and Results

\begin{tabular}{|c|c|c|c|c|c|}
\hline \multirow[t]{2}{*}{ Experiment } & \multirow[t]{2}{*}{ Technique } & \multicolumn{4}{|c|}{$\begin{array}{c}\text { Percentage of supernumerary limbs formed after } \\
\text { surgery at each stage }\end{array}$} \\
\hline & & $\mathrm{DD}$ & MB & LB & $\mathrm{Pal}$ \\
\hline Series 1 & $\begin{array}{l}\text { Rotation of stump skin cuff } \\
\text { at Day } 0 \text { and rerotation of } \\
\text { stump skin cuff and re- } \\
\text { generate epidermis at } \\
\text { various stages. }\end{array}$ & 23.8 & 8.7 & 76.9 & 72.2 \\
\hline Series 2 & $\begin{array}{l}\text { Rotation of stump skin cuff } \\
\text { and regenerate epidermis } \\
\text { at various stages. }\end{array}$ & 75.0 & 84.0 & 85.7 & 70.0 \\
\hline Series 3 & $\begin{array}{l}\text { Rotation of stump skin cuff } \\
\text { at Day } 0 \text { and rerotation of } \\
\text { stump skin cuff at various } \\
\text { stages. }\end{array}$ & - & 66.7 & - & 78.9 \\
\hline Series 4 & $\begin{array}{l}\text { Rotation of stump skin cuff } \\
\text { at various stages of regen- } \\
\text { eration. }\end{array}$ & - & 55.6 & - & 5.9 \\
\hline Series 5 & $\begin{array}{l}\text { Rotation of the epidermis } \\
\text { which covers the regener- } \\
\text { ate at various stages. }\end{array}$ & - & $44.4 / 22.7^{a}$ & - & 81.8 \\
\hline
\end{tabular}

"Indicates the results of two experimental groups in which rotation of epidermis was performed at the stage of medium bud.

1959; Michael and Faber, 1971; Stocum, $1968 \mathrm{a}, \mathrm{b})$. In the blastemal rotation experiments of Iten and Bryant (1975), the anterior-posterior axis of the regenerating limb was found to be specified but unstable at the stage of early bud. These results indicate that cells of very young blastemas possess a certain degree of morphogenetic information. However, the fact that disharmonic morphogenetic instructions can both be corrected in (series 1) and imposed upon (series 2) regenerates which have passed through the early regenerative stages indicates that the morphogenetic information possessed by the early blastema is not irreversibly fixed in these cells. As late as the stage of late bud, at which time cellular condensations have begun to occur in the blastema so that cells destined to become muscle and cartilage are easily distinguished (Tank, 1976; Tank et al., 1976), the regenerate is still recep- tive to the introduction of disharmonic morphogenetic information. The fact that misinformation supplied prior to the stage of late bud cannot be successfully erased by removal of the disruptive influence at that stage demonstrates the tendency of the regenerate toward stable cellular differentiation. Therefore, the stage of late bud appears to be a critical time in regeneration of the axolotl limb during which some cells become determined in the direction of their development.

By the stage of palette, a great deal of cytodifferentiation has occurred (Tank et al., 1976). Cartilaginous primordia of forearm and wrist elements as well as primordia of two or three digits have formed, and muscle primordia are considerably differentiated in proximal regions of the regenerate and less differentiated in more distal regions. In general, the proximal regions of the regenerating limb are the first to 
undergo differentiation (Grim and Carlson, 1974; Michael and Faber, 1971) in a proximo-distal sequence, similar to that observed in avian limb development (Saunders, 1948; Summerbell et al., 1973). Although a great amount of differentiation has occurred in the later stages, the regenerate is still very susceptible to the effects of disharmonic information (scrics 2). These results do not militate against the theory that proximal blastemal structures are determined before distal structures (Faber, 1959; Michael and Faber, 1971; Stocum, 1968b; Tschumi, 1957), but they do demonstrate a plasticity of blastemal cells that has not been shown previously.

These experiments have confirmed the observation (Carlson, 1974, 1975a, b; Lheureux, 1972, 1975a, b) that stump tissues have a great influence on the morphology of the developing regenerate. The earliest time during which significant morphogenetic information transmitted by the stump skin and regenerate epidermis is received by the regenerate and expressed as a multiple structure lies between the medium bud and late bud stages. After this time, the disharmonic morphogenetic information provided by $180^{\circ}$-rotated stump skin has irreversibly influenced the cells of the regenerate, and attempts to correct the disharmony fail.

The regenerate is receptive to morphogenetic input at all stages tested. Although DeBoth (1970) and Stocum (1968a, b) have stated that enough cellular differentiation has occurred by the stage of medium bud to allow differentiation of limb structures to occur in the absence of stump influences, it has been demonstrated that the morphogenetic information possessed by early blastemal cells is not stable. It now seems likely that blastemal cells not only carry morphogenetic information from the region of their origin, but that morphogenetic influences emanate from stump tissues throughout the regenerative process, and that cells are sensitive to the information from the time of their origin as blaste- mal cells. Therefore, it is not until the stage of late bud that some of these undifferentiated cells seem to become irreversibly fixed in their direction of development. Cells which have already "read" morphogenetic instructions prior to the surgical manipulations performed at the stage of late bud may continue along their direction of development, or they may continue "reading" information introduced after that stage. The result is the confused process of pattern formation observed in this study.

Contrary to previous results (series 1), when the disharmonic influence of stump skin is removed at the stage of medium bud by rerotation of the skin independent of the epidermis covering the regenerate, the regenerates continue to produce supernumerary structures. This result could be explained in several ways. After the removal of stump disharmony, the regenerate could have acted, in the sense of Stocum (1968a, b) and DeBoth (1970), as a selforganizing system. In this case, the regenerate would continue to produce supernumerary structures even after the removal of the disharmonic stump stimulus. This seems doubtful in view of the results obtained after a similar duration of disharmonic stimulus was supplicd to the regenerate and was then removed at the stage of medium bud (in series 1). When the epidermis was not rerotated, the stimulus provided by it was sufficient to allow the continued production of supernumerary regenerates, even though stump influences were again in harmony.

The presence of a nonrotated epidermal covering on the regenerate at the stage of medium bud was not sufficient to prevent supernumerary limb production after rotation of the skin of the stump (series 4). In this experimental design, the epidermis was simply another normal influence upon the regenerate which was in harmony with deep stump tissues, but which was in disharmony with the rotated skin. The result was the formation of supernumerary 
limbs. Although the epidermis appears to have exerted a stabilizing influence when stump disharmony was introduced to the regenerate at the stage of palette (series 4), this stability is more likely due to another factor. As stated previously, the regenerate at the stage of palette displays a high level of internal organization and advanced cellular differentiation. It has been shown (Carlson, 1975b; Lheureux, 1972) that stump morphogenetic influences can be exerted over a distance of only 1 or 2 mm through tissues possessing morphogenetic stability. Even though the regenerate is still receptive to morphogenetic input at the stage of palette, the basal regions of the regenerate have become morphogenetically stable by the method described by Grim and Carlson (1974). The effect of rotated stump skin is effectively screened by these stable proximal regions of the blastema.

By the time the regenerate has reached the stage of medium bud, the epidermis that covers the regenerate is well developed, with a clearly defined apical epidermal thickening (AET) (Thornton, 1968). Electron microscopic observation has revealed that the epidermal cells possess a cisternal type of endoplasmic reticulum not seen in nonregenerate epidermis (Singer and Salpeter, 1961). It has been suggested that this type of endoplasmic reticulum may be used for the production of protein-rich substances. Recent evidence (Chapron, 1974) has shown that cells of the AET in the regenerating forelimbs of newts are able to incorporate $\left[{ }^{3} \mathrm{H}\right]$-fucose into an exportable glycoprotein which later appears on the membranes of blastemal cells and even in their cytoplasm. Although no evidence exists which suggests that the product is a morphogenetic messenger, these findings do imply that the epidermis of the regenerate is in very close communication with blastemal tissues during the critical phases of limb regeneration.

The work of Tschumi (1956) on limb de- velopment in Xenopus tadpoles has shown that $90^{\circ}$ rotation of an amphibian ectoderm possessing a distinct apical ectodermal ridge can cause the production of limbs with $90^{\circ}$ axial disturbances. Similarly, the rotation of the epidermis that covers the regenerate (which also possesses an apical thickening) also results in morphogenetic disturbances. When rotation is performed at the stage of medium bud, few supernumerary regenerates of low complexity are formed. 'This indicates that the disharmonious influence causing the regenerative disturbance may be of lesser intensity than the influence responsible for the results of series 1 and 2 . The fact that epidermal rotation at the stage of palette produces regenerates of higher complexity may be due, in part, to the effect of cells accumulating deep to the basement membrane of the epidermis prior to dermis formation. Due to this contamination of the epidermal grafts by blastemal cells, the results of series 5 cannot be attributed entirely to the influence of the epidermis. However, these results do indicate that the epidermis covering the regenerate may influence morphogenesis. This view is in opposition to the results of Glade $(1957,1963)$ and Carlson (1975b). Both Glade and Carlson indicated that stump epidermis does not possess morphogenetic information and suggested that the morphogenetic potential of whole skin resides entirely in the dermis. This may be true for stump tissues, since their experiments involved the rotation of epidermis over stump tissues but not over the regenerate itself. It may be possible for the epidermis covering the regenerate to exert morphogenetic influences on the regenerate merely because it lies in direct contact with the labile cells of the blastema. Although the results of series 5 are not sufficient to disprove the findings of Glade $(1957,1963)$ and Carlson (1975b), they do add a new dimension to the study of morphogenetic interactions in the regenerate.

Whether the role of the amphibian epi- 
dermis is instructive or merely permissive remains a question at this time. The work of Stocum and Dearlove (1972) and Thorton (1968) leaves little doubt that the epidermis is essential to the continued outgrowth of the amphibian limb regenerate. There still remains much work to be done before the role played by the epidermis in the morphogenesis of the regenerating limb is understood.

The present work has revealed that the morphogenetic influence causing the production of multiple structures is not a single pinpoint passage of information. It extends from, at least, the end of the stage of medium bud or the beginning of the stage of late bud (results of series 1) through the stage of palette (results of series 2). The work of Stocum (1968a, b) and DeBoth (1970) indicates that information may be present at even earlier times in the regenerative process. If so, this information does not become stabilized within the blastemal cells until late in the medium bud stage or early in the late bud stage. Some, if not all, blastemal cells are receptive to morphogenetic input as late as the palette stage of regeneration. No conclusions can be drawn, based on these experiments, as to the nature of the morphogenetic message. It does appear, however, that both the stump tissues and the epidermis covering the regenerate supply morphogenetic instructions to the developing blastemal cells. Additional experiments are necessary before definite conclusions can be drawn concerning the role of the epidermis in morphogenesis of the regenerate.

I wish to thank Mr. William Brudon for the original artwork and Ms. Roberta Castro for aid in preparation of the manuscript. I am also grateful to Dr. Bruce Carlson for critical evaluation of the manuscript.

\section{REFERENCES}

Bryant, S. V., and Iten, L. E. (1974). The regulative ability of the limb regeneration blastema of Notophthalmus viridescens: Experiments in situ. Wilhem Roux Arch. Entwicklungsmech. Organismen 174, 90-101.
Carlson, B. M. (1974). Morphogenetic interactions between rotated skin cuffs and underlying stump tissues in regenerating axolotl forelimbs. Devlop. Biol. 39, 263-285.

Carlson, B. M. (1975a). Multiple regeneration from axolotl limb stumps bearing cross-transplanted minced muscle regenerates. Develop. Biol. 45, 203-208.

Carlson, B. M. (1975b). The effects of rotation and positional change of stump tissues upon morphogenesis of the regenerating axolotl limb. Develop. Biol. 47, 269-291.

Chapron, C. (1974). Mise en évidence du rôle, dans la régénération des Amphibiens, d'une glycoprotéine sécrétée par la cape apicale: Étude cytochimique et autoradiographique en microscopie électronique. J. Embryol. Exp. Morphol. 32, 133-145.

DeBoth, N. J. (1970). The developmental potencies of the regeneration blastema of the axolotl limb. Wilhelm Roux Arch. Entwicklungsmech. Organismen 165, 242-276.

Droin, A. (1959). Potentialités morphogènes dans la peau du Triton en régénération. Rev. Suisse Zool. 66, 641-709.

Faber, J. (1959). An experimental analysis of regional organization in the regenerating forelimb of the axolotl (Ambystoma mexicanum). Arch. Biol. 71, 1-72.

Glade, R. W. (1957). The effects of tail tissue on limb regeneration in Triturus viridescens. J. Morphol. 101, 477-521.

Glade R. W. (1963). Effects of tail skin, epidermis and dermis on limb regeneration in Triturus viridescens and Siredon mexicanum. J. Exp. Zool. 152, 169-194.

GrIM, M., and CarLson, B. M. (1974). A comparison of morphogenesis of muscles of the forearm and hand during ontogenesis and regeneration in the axolotl (Ambystoma mexicanum). II. The development of muscular pattern in the embryonic regenerating limb. Z. Anat. Entwicklungs. gesch. 145, 149-167.

ITEN, L. E., and Bryant, S. V. (1975). The interaction between the blastema and stump in the establishment of the anterior-posterior and proximal distal organization of the limb regenerate. Develop. Biol. 44, 119-147.

Lheureux, E. (1972). Contribution a l'étude du rôle de la peau et des tissus axiaux du membre dans le déclenchement de morphogèneses régénératrices anormales chez le triton Pleurodeles waltlii Michah. Ann. Embryol. Morphog. 5, 165-178.

Lheureux, E. (1975a). Nouvelles données sur les rôles de la peau et des tissus internes dans la régénération du membre du triton Plearudeles waltlii Michah (Amphibien Urodèle). Wilhelm Roux Arch. Entwicklungsmech. Organismen 176, 285-301.

Lhe UREUX, E. (1975b). Régénération des membres 
irradiés de Pleurodeles waltlii Michah (Urodèle) Influence des qualites et orientations des greffons non irradies. Wilhelm Roux Arch. Entwicklungsmech. Organismen 176, 303-327.

Michael, M. L., and Faber, J. (1971). Morphogenesis of mesenchyme from regeneration blastemas in the absence of digit formation in Ambystoma mexicanum. Wilhelm Roux Arch. Entwicklungsmech. Organismen 168, 174-180.

Rahmani, T. (1960). Conflit de potentialités morphogènes et duplicare. Reu. Suisse Zool. 67, 589675.

Saunders, J. W., JR. (1948). The proximo-distal sequence of origin of the parts of the chick wing and the role of the ectoderm. J. Exp. Zool. 108, 363-403.

Setrles, H. E. (1967). Supernumerary regeneration caused by ninety degree skin rotation in the adult newt, Triturus viridescens. Ph.D. Thesis, Tulane University.

SetTles, H. E. (1970). Morphogenetic effects of limb skin during limb regeneration in the adult newt, Triturus viridescens. (Abstract) Anat. Rec. 166, 375.

Singer, M., and Salpeter, M. M. (1961). Regeneration in vertebrates: The role of the wound epithelium. In "Growth in Living Systems" (M. X. Zarrow, ed.), pp. 277-311. Basic Books, New York.

Stocum, D. L. (1968a). The urodele limb regeneration blastema: A self-organizing system. I. Differentiation in vitro. Develop. Biol. 18, 441-456.
Stocum, D. L. (1968b). The urodele limb regeneration blastema: A self-organizing system. II. Morphogenesis and differentiation of autografted whole and fractional blastemas. Develop. Biol. 18, 457-480.

Stocum, D. L., and Dearlove, G. E. (1972). Epidermal-mesodermal interaction during morphogenesis of the limb regeneration blastema in larral salamanders. J. Exp. Zool. 181, 49-62.

Summerbell, D., Lewis, J. H., and Wolpert, L. (1973). Positional information in chick limb morphogenesis. Nature (London) 244, 492-496.

Tank, P. W. (1976). The timing of morphogenetic events in the regenerating forelimb of the axolotl, Ambystoma mexicanum. Ph.D. Thesis, The University of Michigan.

Tank, P. W., Carlson, B. M., and Conelly, T. G. (1976). A staging system for forelimb regeneration in the axolotl, Ambystoma mexicanum. J. Morphol. 150, 117-128.

Thorton, C. S. (1968). Amphibian limb regeneration. In "Advances in Morphogenesis" (M. Abercrombie, J. Brachet and T. J. King, eds.), Vol. 7, pp. 205-249. Academic Press, New York.

'I'schumi, P. A. (1956). Die bedeutung der epidermisleiste für die entwicklung der beine von Xenopus laevis Daud. Rev. Suisse Zool. 63, 707-716.

Tschumi, P. A. (1957). The growth of the hindlimb bud of Xenopus laevis and its dependence upon the epidermis. J. Anat. 91, 149-173. 